\title{
The Cell-Autonomous Clock of VIP Receptor VPAC2 Cells Regulates Period and Coherence of Circadian Behavior
}

\author{
${ }^{\circledR}$ Ryan Hamnett, ${ }^{\circledR}$ Johanna E. Chesham, ${ }^{\circledR}$ Elizabeth S. Maywood, and ${ }^{\circledR}$ Michael H. Hastings \\ Division of Neurobiology, MRC Laboratory of Molecular Biology, Cambridge, Cambridgeshire CB2 0QH, United Kingdom
}

Circadian (approximately daily) rhythms pervade mammalian behavior. They are generated by cell-autonomous, transcriptional/translational feedback loops (TTFLs), active in all tissues. This distributed clock network is coordinated by the principal circadian pacemaker, the hypothalamic suprachiasmatic nucleus (SCN). Its robust and accurate time-keeping arises from circuit-level interactions that bind its individual cellular clocks into a coherent time-keeper. Cells that express the neuropeptide vasoactive intestinal peptide (VIP) mediate retinal entrainment of the SCN; and in the absence of VIP, or its cognate receptor VPAC2, circadian behavior is compromised because SCN cells cannot synchronize. The contributions to pace-making of other cell types, including VPAC2-expressing target cells of VIP, are, however, not understood. We therefore used intersectional genetics to manipulate the cell-autonomous TTFLs of VPAC2-expressing cells. Measuring circadian behavioral and SCN rhythmicity in these temporally chimeric male mice thus enabled us to determine the contribution of VPAC2-expressing cells ( $\sim 35 \%$ of SCN cells) to SCN time-keeping. Lengthening of the intrinsic TTFL period of VPAC2 cells by deletion of the $C K 1 \varepsilon^{\mathrm{Tau}}$ allele concomitantly lengthened the period of circadian behavioral rhythms. It also increased the variability of the circadian period of bioluminescent TTFL rhythms in SCN slices recorded ex vivo. Abrogation of circadian competence in VPAC2 cells by deletion of Bmall severely disrupted circadian behavioral rhythms and compromised TTFL time-keeping in the corresponding SCN slices. Thus, VPAC2-expressing cells are a distinct, functionally powerful subset of the SCN circuit, contributing to computation of ensemble period and maintenance of circadian robustness. These findings extend our understanding of SCN circuit topology.

Key words: bioluminescence; Bmal1; casein kinase; circadian rhythm; neuropeptide; suprachiasmatic nucleus

Significance Statement

Circadian (approximately daily) rhythms govern our lives. They are coordinated by the brain's central clock, the suprachiasmatic nucleus (SCN) of the hypothalamus. The SCN works so well because of powerful interactions between its $\sim 20,000$ cells. But SCN cells are very heterogeneous, and the specific time-keeping contributions of particular subgroups are unknown. Using mice, we genetically manipulated the clock of cells expressing VPAC2, the receptor for an important SCN neuropeptide: VIP. Slowing down VPAC2 cellular clocks slowed down mouse behavioral rhythms. Stopping the clock of VPAC2 cells disrupted circadian behavior and SCN rhythms. These findings extend the understanding of SCN circuitry by showing that VPAC2-expressing cells are a distinct, functionally powerful component of the SCN circuit, governing its period and robustness.

Received July 30, 2020; revised Sep. 28, 2020; accepted 0ct. 6, 2020.

Author contributions: R.H., E.S.M., and M.H.H. designed research; R.H. and J.E.C. performed research; R.H. analyzed data; R.H. wrote the first draft of the paper; R.H., E.S.M., and M.H.H. edited the paper; R.H. and M.H.H. wrote the paper.

This work was supported by the Medical Research Council MC_U105170643 core funding to M.H.H. We thank the staff of the Medical Research Council LMB Biomedical Facility, Ares, for mouse breeding and handling; the staff of the Light Microscopy Facility for imaging support; and Emma Morris for experimental assistance.

R. Hamnett's present address: Department of Neurosurgery, Stanford University, Stanford, CA 94305.

The authors declare no competing financial interests.

Correspondence should be addressed to Michael H. Hastings at mha@mrc-Imb.cam.ac.uk or Ryan Hamnett at rhamnett@stanford.edu.

https://doi.org/10.1523/JNEUROSCI.2015-20.2020

Copyright $\odot 2021$ Hamnett et al.

This is an open-access article distributed under the terms of the Creative Commons Attribution 4.0 International license, which permits unrestricted use, distribution and reproduction in any medium provided that the original work is properly attributed.

\section{Introduction}

Mammalian physiology and behavior are rhythmic and adaptively aligned with the environmental light-dark cycle by the suprachiasmatic nucleus ( $\mathrm{SCN}$ ) of the hypothalamus, which is entrained by direct retinal innervation (Reppert and Weaver, 2002). Within the 20,000 "clock" cells of the SCN, and indeed most cells across the body, cell-autonomous circadian timekeeping is maintained by a transcriptional/translational feedback loop (TTFL) in which CLOCK:BMAL1 heterodimers activate transcription of Period1/2 and Cryptochrome1/2 genes, the protein products of which (PER1/2 and CRY1/2) feedback to repress their own transcription. This cycle takes $\sim 24 \mathrm{~h}$ to complete, although SCN period can be modified considerably by genetic or pharmacological manipulation (Patton et al., 2016), such as the 
Tau mutation in casein kinase $1 \varepsilon\left(\mathrm{CK} 1 \varepsilon^{\mathrm{Tau}}\right)$, which shortens it to $20 \mathrm{~h}$ in homozygous mice (Meng et al., 2008). More dramatically, mice lacking BMAL1 (Bmal1 $\left.{ }^{-1-}\right)$, the only nonredundant TTFL component, are arrhythmic at cellular and behavioral levels (Bunger et al., 2000): effects that are reversible by transgenic rescue (McDearmon et al., 2006).

Beyond the cell-autonomous clock, SCN pace-making is a product of circuit-level interactions that bind the circadian cycles of the individual cells. These interactions confer onto the network its essential emergent properties of robust, high-amplitude and synchronized cellular oscillations, with established ensemble phase and period (Hastings et al., 2018). Indeed, the power of intercellular coupling can partially compensate for various genetic losses within the TTFL (Liu et al., 2007; Ko et al., 2010). Although GABA is the principal neurotransmitter of the SCN, the strongest evidence for neurochemical mediation of intercellular coupling is not for GABAergic signaling but, rather, for a hierarchy of neuropeptides (Maywood et al., 2011). Across the SCN, discrete populations of cells are characterized by their expression of, inter alia, vasoactive intestinal peptide (VIP), arginine vasopressin (AVP), gastrinreleasing peptide (GRP), prokineticin2 (Prok2), and their cognate receptors (Abrahamson and Moore, 2001; Antle and Silver, 2005; Park et al., 2016). These cell types show a highly stereotypical spatial organization within the SCN, with VIP and GRP cells in the retinorecipient "core," and AVP and Prok2 cells in its surrounding "shell." Mice or SCN slices deficient in intercellular communication mediated by VIP and its receptor VPAC2 (encoded by the Vipr2 gene), display weakened rhythmicity, fewer rhythmic neurons, and damped and desynchronized cellular oscillations (Harmar et al., 2002; Colwell et al., 2003; Aton et al., 2005; Maywood et al., 2006; Ciarleglio et al., 2009). Moreover, VIP cells receive the retinal information that entrains the SCN to solar time (Abrahamson and Moore, 2001; Jones et al., 2018; Mazuski et al., 2018). In turn, VIP acts via the VPAC2-expressing cells of the SCN shell to maintain steady-state circuit-level coherence and to reset ensemble phase in response to retinal input. It achieves this via a cascade of kinase-dependent signaling (including extracellular signal-regulated kinase (ERK) $1 / 2$ and its regulator, dual specificity phosphatase (DUSP)4) and consequent regulation of a broad transcriptional network (Hamnett et al., 2019). VIP is thereby able to control both cell-autonomous and circuit-level circadian oscillations within the SCN.

The VIP/VPAC2 axis is therefore a central element of SCN circuit topology. Although some functions of VIP cells are established, the functions of their target cells expressing VPAC2, which constitute the next step in the SCN synaptic circuitry, are not. Located in the SCN shell, they may mediate circadian output from the SCN, and/or they may contribute to the circuit-level computations that generate its emergent properties. To investigate this, we used transgenic mice in which VPAC2 cells express Cre recombinase (Patton et al., 2020). This allowed conditional manipulation of the cell-autonomous TTFL of VPAC2-expressing cells, altering their intrinsic period by deletion of $C k 1 \varepsilon^{\text {Tau }}$, or their circadian competence by deletion of Bmal1. By monitoring the consequences for behavior and SCN pace-making, we reveal that VPAC2-expressing cells are a distinct, functionally powerful subset of the SCN circuit, contributing to computation of ensemble period and maintenance of robustness. These findings extend our understanding of SCN circuit topology.

\section{Materials and Methods}

Animals. All animals were cared for in accordance with the UK Animals (Scientific Procedures) Act of 1986 with local ethical approval (LMB Animal Welfare and Ethical Review Body). VPAC2-Cre mice (Tg (Vipr2cre)KE2Gsat/Mmucd; RRID:MMRRC_034281-UCD) were purchased from GENSAT (Gene Expression in the Nervous System Atlas) project (Rockefeller University). These mice were subsequently crossed with either $C k 1 \varepsilon^{\mathrm{Tau} / \mathrm{Tau}}$ (Meng et al., 2008) or Bmall ${ }^{f l f / f l x}$ mice (generated from The Jackson Laboratory, mouse stock \#007668, RRID:IMSR JAX:007668). Both the Ck1 $\varepsilon^{\text {Tau/Tau }}$ and Bmalf ${ }^{f l / f l x}$ mice contain floxed exons that can be removed through Cre-mediated recombination. Because of VPAC2-Cre expression in developing spermatocytes (Usdin et al., 1994; Krempels et al., 1995), which is a known issue with some Cre driver lines (Luo et al., 2020), recombination occurred before fertilization, resulting in offspring containing one recombined allele in all cells, alongside the remaining floxed allele to be deleted only in somatic cells expressing VPAC2-Cre. These crosses, therefore, generated VPAC2-Cre/Ck1 $\varepsilon^{\text {Tau/- }}$ and VPAC2-Cre/Bmal1 ${ }^{f l x /-}$ mice. Heterozygote WT/heterozygote null mice for $C k 1 \varepsilon\left(C k 1 \varepsilon^{W T /-}\right)$ and Bmal1 (Bmal1 ${ }^{W T /-}$ ) were therefore used as controls. All mice were also crossed to PER2::LUCIFERASE knock-in mice (gift from Prof Joseph Takahashi, University of Texas Southwestern Medical Center) (Yoo et al., 2004) for visualization of circadian dynamics through bioluminescent recording, and R26R-EYFP mice (The Jackson Laboratory, stock \#006148, RRID:IMSR_JAX:006148) to report Cre-mediated recombination and determine Bmal1 deletion efficiency (Srinivas et al., 2001). Finally, crossing dopamine 1A-receptor (Drd1a)-Cre mice (GENSAT, RRID: MMRRC_030779-UCD) with Bmal1 ${ }^{f l x /}$ mice generated Drdla-Cre/ $B_{\text {mall }}^{f l x /-}$ mice. This Cre line has previously been shown to have extensive expression in the SCN, covering $63 \%$ of SCN cells and colocalizing with $62 \%$ of AVP cells and $81 \%$ of VIP cells (Smyllie et al., 2016). The Drdla-Cre population has also been shown as being capable of dictating period by crossing with $C k 1 \varepsilon^{\text {Tau/Tau }}$ mice. Given its expression profile, it served here as a comparator control for Bmall deletion in VPAC2-Cre cells.

Mouse wheel-running behavior and analysis. Because the circadian behavior of adult female mice is modulated by the estrous cycle, and the estrous cycle is itself a product of SCN circadian timekeeping, this project used only male mice to avoid potential indirect effects on behavior arising from SCN-estrous-behavior interactions. Male mice were individually housed and kept in a ventilated stainless-steel cabinet with controlled lighting for the duration of behavioral monitoring. Their activity patterns were assessed using running wheels (ActiMetrics) alongside passive infrared movement detectors. Mice were typically entrained to a cycle of $12 \mathrm{~h}$ light ( $\mathrm{L}, \sim 200 \mathrm{lux})$ and $12 \mathrm{~h}$ dim red light (D, <10 lux) $(12: 12 \mathrm{LD})$ for at least $7 \mathrm{~d}$ to assess entrainment to $24 \mathrm{~h}$ rhythms, before being transferred to constant dim red light (DD) to investigate free-running period. Food and water were provided ad libitum. Wheel revolutions and general movement data were acquired and stored in $6 \mathrm{~min}$ bins. Data were analyzed using ClockLab version 6 (ActiMetrics; RRID: SCR_014309) with behavioral circadian period in different lighting conditions determined by $\chi^{2}$ periodogram. The emergence of the arrhythmic/disordered phenotype in Bmall-deleted mice was determined by eye. The robustness of the rhythms of such mice was quantified using the relative amplitude (RA) nonparametric measure in ClockLab version 6 on the final $14 \mathrm{~d}$ of DD recording.

SCN organotypic slices: bioluminescent recordings and analysis. SCN organotypic slices and media formulations were prepared as described by Hastings et al. (2005). Briefly, brains were dissected from adult mice and placed into ice-cold dissection medium. SCN tissue was isolated from $300 \mu \mathrm{m}$ slices prepared using a McIlwain Tissue Chopper (RRID: SCR_015798) and cultured on a Millicell filter membrane (Millipore, RRID:SCR_015799) in $1 \mathrm{ml}$ culture medium. SCN slices acclimatized for $3-6 \mathrm{~h}$ at $37^{\circ} \mathrm{C}, 5 \% \mathrm{CO}_{2}$, and were then transferred to $35 \mathrm{~mm}$ culture dishes containing $1.2 \mathrm{ml}$ recording medium and sealed with glass coverslips, secured with silicon grease, for bioluminescent recordings. Slices were placed under photomultiplier tubes (PMTs; H9319-11 photoncounting head, Hamamatsu) in a light-tight incubator kept at $37^{\circ} \mathrm{C}$ for recording of bioluminescence. These recordings were analyzed to 
Table 1. Primary antisera used for immunohistochemistry

\begin{tabular}{lllll}
\hline Name & Host & Supplier and catalog number & RRID & Concentration \\
\hline Anti-AVP & Rabbit & Peninsula T-4563 & AB_518673 & $1: 1000$ \\
Anti-Bmal1 & Rabbit & In-house (Reddy et al., 2006) & NA & $1: 500$ \\
Anti-GRP & Rabbit & Immunostar 20073 & AB_572221 & $1: 1000$ \\
Anti-VIP & Rabbit & Immunostar 20077 & AB_572270 & $1: 750$ \\
\hline
\end{tabular}

calculate circadian period, amplitude and RA error (RAE; a measure of the rhythm robustness) using the FFT-nonlinear least squares function in the BioDare2 software (Zielinski et al., 2014). A $24 \mathrm{~h}$ rolling average subtraction was performed on individual traces to account for variable baselines. The first $12 \mathrm{~h}$ of recordings was not included in the analysis to exclude potential artifacts arising from slice preparation. To track the progression of period change in $C k 1 \varepsilon^{T a u}$ experiments, the time between successive PER2::LUCIFERASE peaks was determined.

Immunohistochemistry and image analysis. For histologic analysis, adult mice were killed rapidly by cervical dislocation, and the brains dissected and immediately postfixed in $10 \mathrm{ml} 4 \%$ paraformaldehyde (PFA) (Alfa Aesar) in $0.1 \mathrm{M}$ phosphate buffer (PB) for $4-5 \mathrm{~h}$ at room temperature, before being cryopreserved in $20 \%$ sucrose (Fisher Chemical) in phosphate buffered saline (PBS) at $4^{\circ} \mathrm{C}$ overnight. Coronal sections $(40 \mu \mathrm{m})$ were taken using a freezing microtome (Anglia Scientific), incubated for $1 \mathrm{~h}$ at room temperature in 2\% normal serum in PBS with $1 \%$ bovine serum albumin (BSA) and $0.3 \%$ Triton X-100, and then transferred to primary antisera incubation overnight at $4^{\circ} \mathrm{C}$ (for antisera details, see Table 1). Tissue was then incubated with appropriate secondary antibodies (1:500) for $1 \mathrm{~h}$ at room temperature. Sections were mounted onto slides and coverslipped using Vectashield Hardset mounting medium with DAPI (Vector Labs, RRID:AB_2336788).

Fluorescence imaging was conducted using 710 and 780 confocal microscopes (Carl Zeiss). Whole SCN sections were imaged using a $20 \times$ air objective (numerical aperture (NA) 0.5 or 0.8 on the 710 and 780 microscopes, respectively) while more detailed images (required for cellcounting analysis) were acquired with a $63 \times$ oil objective, NA 1.4, before subsequent automated tile-stitching was performed by the Carl Zeiss software (Zen 9 or 10). Counts of cells in SCN sections and determination of fluorescence intensity of SCN neuropeptides were performed in Fiji.

Experimental design and statistical analysis. Statistical tests and graphical representation of data (mean \pm SEM) were performed using Prism 6 and 7 software (GraphPad). Statistical comparisons were performed using one- or two-way ANOVA with Tukey's multiple comparisons correction, unless otherwise stated. Kruskal-Wallis tests were performed when comparing RA scores between genotypes followed by Dunn's correction for multiple comparisons. Correlation was determined using Pearson's correlation coefficient. Mice and SCN slices were assigned randomly, without regard to genotype, to activity-recording cages and to photomultiplier recording systems, respectively. For both mice in vivo and SCN slices ex vivo, procedures were performed simultaneously on all genotypic groups within an experimental cohort.

\section{Results}

\section{VPAC2 cells determine the period of the in vivo circadian} rhythm of wheel-running behavior

The period-setting potential of VPAC2 cells was examined by generating temporally chimeric mice using intersectional genetics to delete the Tau allele of the $C k 1 \varepsilon$ gene in VPAC2-expressing cells. $C k 1 \varepsilon^{T a u}$ is a semidominant point mutation that causes a gain of function for the CK1 $\varepsilon$ protein that shortens the WT behavioral period by $2 \mathrm{~h}$ per copy (heterozygote: $22 \mathrm{~h}$ period; homozygote: $20 \mathrm{~h}$ ) (Meng et al., 2008). In contrast, mice lacking $\mathrm{CK} 1 \varepsilon\left(C k 1 \varepsilon^{-1-}\right)$, following Cre-mediated excision of a floxed exon carrying the mutation, have a $24 \mathrm{~h}$ period, whereas $C k 1 \varepsilon^{\mathrm{Tau} /-}$ exhibit a $22 \mathrm{~h}$ period. Hence, in the VPAC2-Cre/Ck1 $\varepsilon^{\text {Tau/- }}$ mouse, VPAC2-Cre cells would have a period of $24 \mathrm{~h}$ (being $C k 1 \varepsilon^{-/-}$), whereas the rest of the SCN and other tissues would retain a period of $22 \mathrm{~h}$ (Fig. 1A). If the cell-autonomous TTFL of VPAC2 cells directs circadian behavior, such lengthening of their intrinsic period should correspondingly lengthen the period of wheel-running rhythms of the mouse.

The wheel-running behavior of mice of all genotypes $\left(C k 1 \varepsilon^{W T /-}\right.$ and $C k 1 \varepsilon^{T a u /}$, without and with VPAC2-Cre) entrained stably to a 12:12 light:dim red light (LD) cycle (Fig. $1 B, C$; two-way ANOVA, $F_{(1,18)}=3.706, p=0.07$, Cre; $F_{(1,18)}=0.000, p>0.999$, Tau; $F_{(1,18)}=$ $0.000, p>0.999$, interaction). On transfer to continuous dim red light (DD), $C k 1 \varepsilon^{W T /-}$ mice free-ran with a WT equivalent period $(\sim 24 \mathrm{~h})$, whereas that of $C k 1 \varepsilon^{\mathrm{Tau} /-}$ mice was significantly shorter ( $\sim 22$ h, Fig. $1 B, D$; two-way ANOVA, $F_{(1,39)}=30.46, p<0.0001$, interaction; Tukey's post hoc test, $p<0.0001)$. The presence of VPAC2-Cre had no effect in $C k 1 \varepsilon^{W T /-}$ mice lacking the floxed allele, whereas its presence in the $C k 1 \varepsilon^{\text {Tau/- }}$ mice caused significant lengthening of circadian period (Tukey's post hoc test $C k 1 \varepsilon^{\text {Tau/ }-}, 22.21 \pm 0.08 \mathrm{~h}$ vs VPAC2-Cre/Ck1 $\varepsilon^{\text {Tau/- }}$, $23.49 \pm 0.12 \mathrm{~h}, p<0.0001)$. This VPAC2-Cre/Ck1 $\varepsilon^{\text {Tau/- }}$ group mean was slightly, albeit significantly, below the $24 \mathrm{~h}$ period of $C k 1 \varepsilon^{W T /-}$ controls, with or without VPAC2-Cre (Tukey's post hoc test $C k 1 \varepsilon^{W T /-}$ vs VPAC2-Cre/Ck1 $\left.\varepsilon^{\text {Tau/- }} p=0.004\right)$. These results were consistent with concomitant determination of period when assessed by passive infra-red movement detectors $\left(C k 1 \varepsilon^{W T /-}\right.$ : $24.06 \pm 0.07 \mathrm{~h}$; VPAC2-Cre/Ck1 $\varepsilon^{W T /-}: 24.08 \pm 0.08 \mathrm{~h} ; C k 1 \varepsilon^{\text {Tau/- }}$ : $22.26 \pm 0.11 \mathrm{~h}$; VPAC2-Cre/Ck1 $\varepsilon^{\mathrm{Tau} /}$ : $\left.23.44 \pm 0.09 \mathrm{~h}\right)$. Nevertheless, at a behavioral level, the cell-autonomous clock of VPAC2 cells can exert a clear influence on circadian period, lengthening it in line with their intrinsic period. Furthermore, the coherence and amplitude of the behavioral rhythm were not affected by temporal chimerism (Fig. 1E; Kruskal-Wallis test, $\mathrm{H}(3)=3.661, p=0.30$ ), confirming that circuit-level mechanisms are able to sustain coherent circadian output in the face of widely divergent cell-autonomous periods in the SCN (Smyllie et al., 2016; Brancaccio et al., 2017).

To test further the effect of chimerism on SCN integrity, sections of adult mouse brain were processed for immunohistochemical staining of the neuropeptides VIP, AVP, and GRP. The morphology of the SCN was comparable across all genotypes, and there were no significant differences in the expression of VIP (two-way ANOVA, $F_{(1,9)}=0.001, p=0.97$, Cre; $F_{(1,9)}=0.628$, $p=0.45$, Tau; $F_{(1,9)}=0.518, p=0.49$, interaction) or GRP (Fig. $1 F$, $G$; two-way ANOVA, $F_{(1,9)}=0.016, p=0.90$, Cre; $F_{(1,9)}=0.000$, $p=0.99$, Tau; $F_{(1,9)}=0.062, p=0.81$, interaction). Compared with $C k 1 \varepsilon^{\text {WT/- }}$ controls, however, the $C k 1 \varepsilon^{\text {Taul- }}$ mice showed a small ( $\sim 20 \%$ ) but significant reduction of AVP expression (Fig. 1F,G; two-way ANOVA, $F_{(1,9)}=0.013, p=0.91$, Cre; $F_{(1,9)}=5.751$, $p=0.04, \mathrm{Tau} ; F_{(1,9)}=0.053, p=0.82$, interaction). This was evident in SCN from mice both without and with the VPAC2-Cre and so could not be a direct cause of the period lengthening observed in the latter. Temporal chimerism did not, therefore, affect the structural integrity of the SCN, nor the quality of behavioral circadian output, and revealed the pace-setting ability of SCN VPAC2 cells.

\section{Period determination by VPAC2 cells is attenuated in the SCN ex vivo}

Following the recording of wheel-running behavior, mice were killed under dim red light and their SCN PER2-driven bioluminescence rhythms were recorded to determine the impact of temporal chimerism on the intrinsic SCN TTFL. SCN of all four genotypes exhibited robust and coherent bioluminescent rhythms (Fig. 2A). As with behavioral periods, the rhythms were shortened by $\sim 2 \mathrm{~h}$ by the presence of a single copy of the Tau allele in the SCN of mice lacking VPAC2-Cre (Fig. 2A,B; 
A

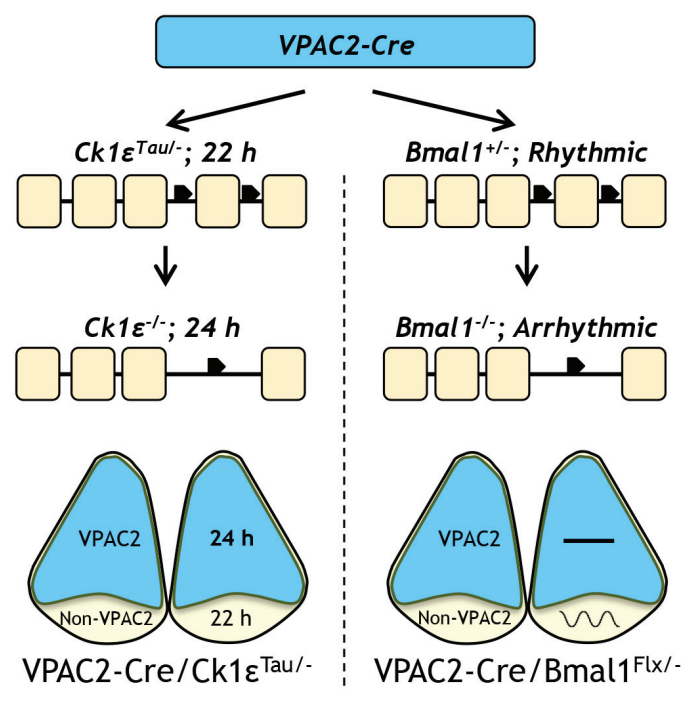

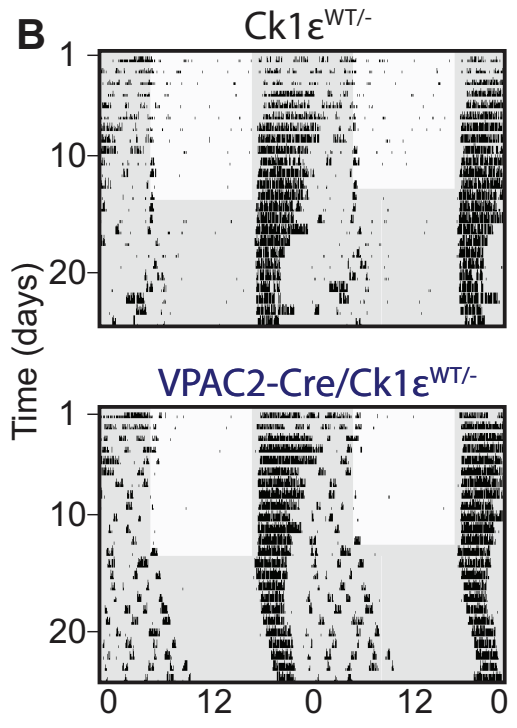

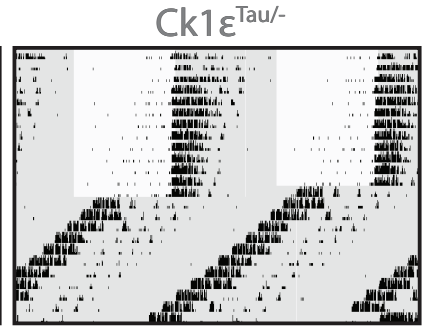

Time of day (hours)

$E$
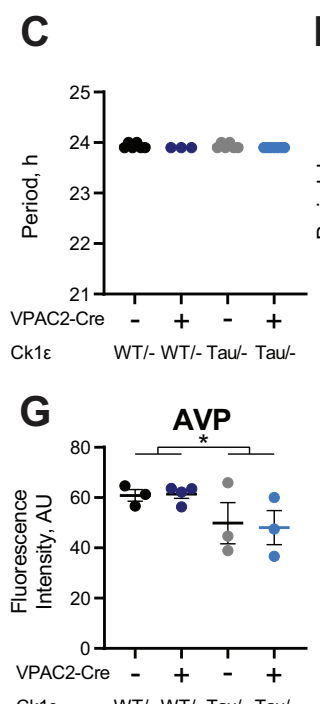
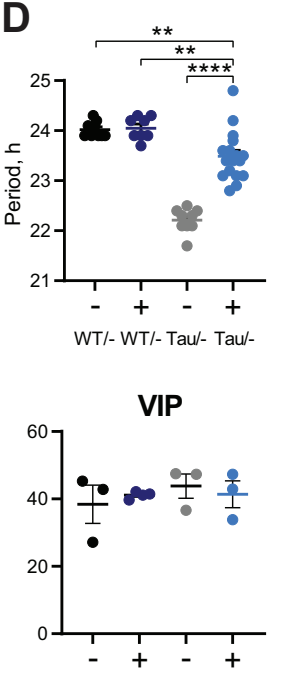

WT/- WT/- Tau/- Tau/-

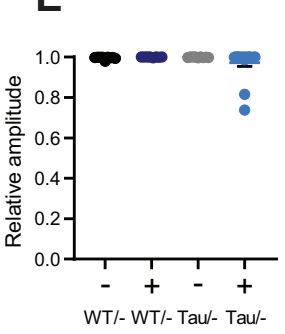

GRP

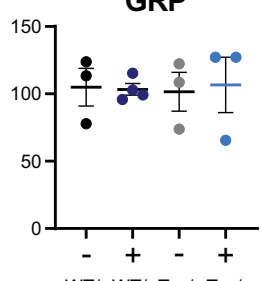

WT/-WT/- Taw/- Taw/-
$\mathbf{F}$

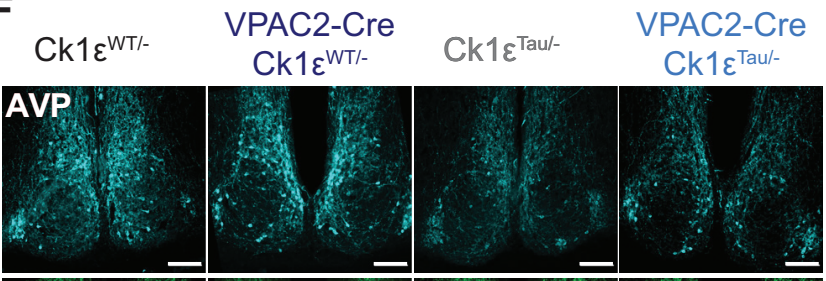

Figure 1. Intersectional genetics reveals that VPAC2-expressing cells determine the circadian period of mouse wheel-running behavior. $\boldsymbol{A}$, Schematic view of the intersectional approach, whereby mice carrying Cre recombinase as a transgene controlled by the VPAC2 promoter were crossed with either $C k 1 \varepsilon^{\text {Tau/- }}$ or Bmall ${ }^{\text {flx/- }}$ mice to create a chimeric SCN. The result is for VPAC2 cells to lack either the Tau allele or Bmal1, reverting them to a $24 \mathrm{~h}$ period as $C \mathrm{k}_{1} \varepsilon^{-1-}$ or rendering them arrhythmic as Bmal1 ${ }^{-1-}$, respectively. $\boldsymbol{B}$, Representative double-plotted actograms of wheel-running activity of VPAC2-Cre/Ck1 $\varepsilon^{\text {Tau/- }}$ mice and their respective controls exposed to a 12:12 light/dim red light (LD) cycle followed by continuous dim red light (DD) conditions. Gray shading represents dim red light. C, Periods (mean \pm SEM) observed in 12:12 LD: $n=6\left(C k 1 \varepsilon^{\text {WT/- }}\right), n=3\left(\mathrm{VPAC}-\mathrm{Cre} / \mathrm{Ck} 1 \varepsilon^{\text {WT/-}}\right), n=6\left(\mathrm{Ck} 1 \varepsilon^{\text {Tau/- }}\right)$, and $n=7\left(\mathrm{VPAC}-\mathrm{Cre} / \mathrm{Ck} 1 \varepsilon^{\text {Tau/-}}\right)$. D, Periods (mean $\left.\pm \mathrm{SEM}\right)$ observed in DD ( $n=9, n=8, n=9, n=17)$ of VPAC2-Cre/Ck1 $\varepsilon^{\text {Tau/- }}$ mice and their respective controls. $\boldsymbol{E}$, RA scores (mean \pm SEM) from $10 \mathrm{~d}$ of wheel-running activity under DD ( $n$ values as in D). Kruskal-Wallis test. $\boldsymbol{F}$, Representative images of immunohistochemical staining of SCN of VPAC2-Cre/Ck1 $\varepsilon^{\text {Tau/- }}$ mice and their respective controls for AVP-ir (top), VIP-ir (middle), and GRP-ir (bottom). Scale bars, $100 \mu \mathrm{m}$. G, Fluorescence intensities (mean \pm SEM) for immunohistochemical staining of AVP-ir (left), VIP-ir (middle), and GRP-ir (right) in SCN sections of VPAC2-Cre/Ck1 $\varepsilon^{\text {Tau/- }}$ mice with their respective controls ( $n=3$ per genotype). $* p<0.05 ; * * p<0.01$; $* * * * p<0.0001$; two-way ANOVAs (main effects of Cre and Tau allele) with Tukey's post hoc test.

two-way ANOVA, $F_{(1,21)}=39.01, p<0.0001$, Tau). In contrast to the lengthening of period observed in vivo, however, there was no systematic or significant effect of VPAC2-Cre on the mean circadian period of $C k 1 \varepsilon^{\text {Tau/- }}$ SCN slices (two-way ANOVA, $F_{(1,21)}=0.408, p=0.53$, interaction), and the mean circadian period was not statistically different from $C k 1 \varepsilon^{T a u /-}$ slices lacking VPAC2-Cre. There was, however, a marked variability within the VPAC2-Cre/Ckl $1 \varepsilon^{\text {Tau/- }}$ group, with a range of $\sim 6.3 \mathrm{~h}$, a clear departure from the behavioral data (Fig. $2 B, C$ ). Furthermore, in the control groups, there was a direct correspondence between the circadian periods measured in vivo as behavior and ex vivo as SCN bioluminescence within individual mice, with slices typically having a slightly longer period (Fig. $2 C)$. This was not the case for the VPAC2-Cre/Ck1 $\varepsilon^{\text {Taul- }}$ group, in which the ex vivo SCN periods were not only widely divergent, but were also not consistent with the in vivo period of the corresponding mouse, typically being shorter (Fig. 2C; two-way ANOVA, $F_{(3,19)}=8.975, p=0.0007$, interaction; Sidak's post hoc $p<0.0001, \mathrm{VPAC} 2-\mathrm{Cre} / C k 1 \varepsilon^{\mathrm{Tau} /-}$, behavior vs slice period), and comparable to or even faster than those of $C k 1 \varepsilon^{\text {Tau/- }}$ SCN lacking recombinase. Consequently, when in vivo and ex vivo periods were plotted together, the control groups exhibited a highly significant within-animal correlation, but the VPAC2-Cre/Ck1 $\varepsilon^{\mathrm{Tau} /-}$ group did not (Fig. 2D; Pearson correlation, Controls: $r=0.923, p<0.0001$, VPAC2-Cre/ $\left.C k 1 \varepsilon^{\text {Tau/- }}: r=0.598, p=0.07\right)$.

The period lengthening by deletion of $C k 1 \varepsilon^{T a u}$ in VPAC2 cells was therefore consistently effective in vivo but had variable 
A $\mathrm{Ck} 1 \varepsilon^{\mathrm{WT} /}$

VPAC2-Cre/Ck1 $\varepsilon^{\mathrm{WT} /-}$ Ck1 $\varepsilon^{\text {Taul- }}$

VPAC2-Cre/Ck1 $1 \varepsilon^{\text {Taul- }}$

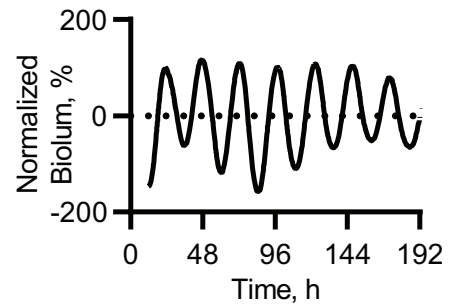

B

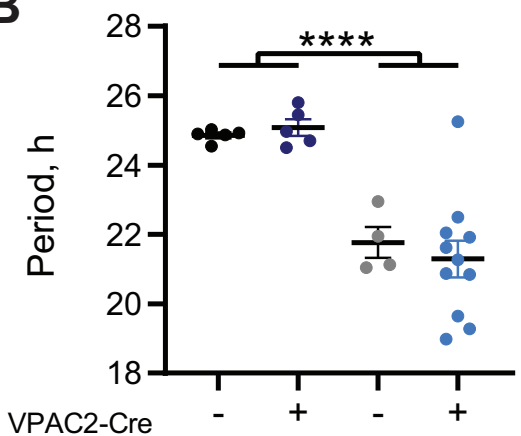

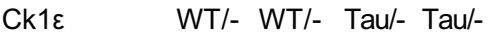

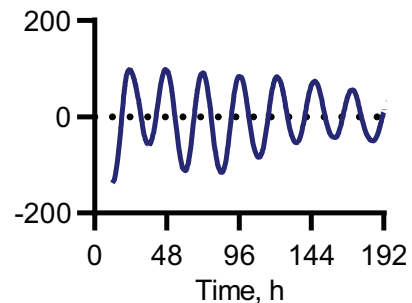

C
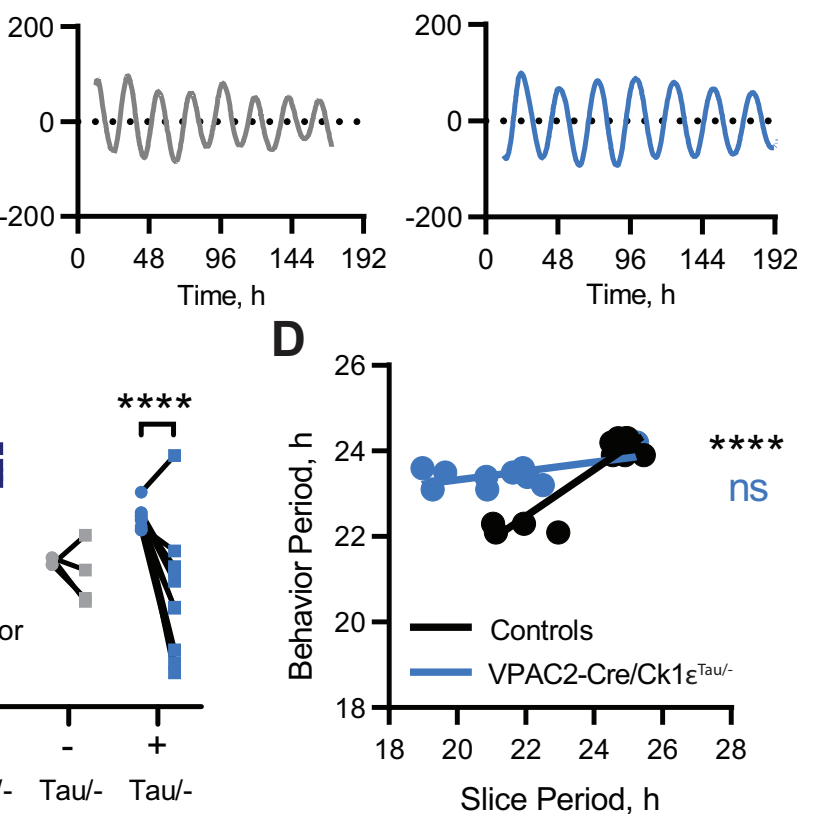

E

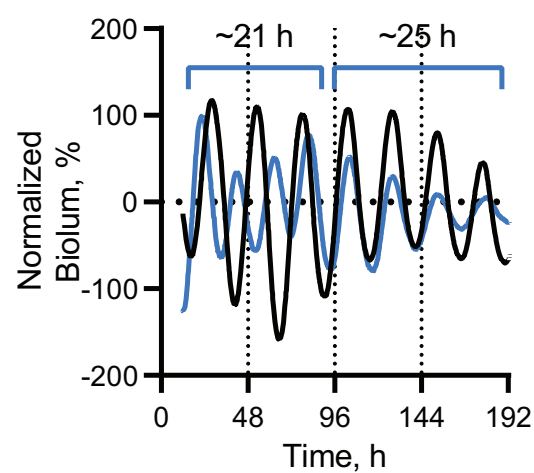

$\mathbf{F}$

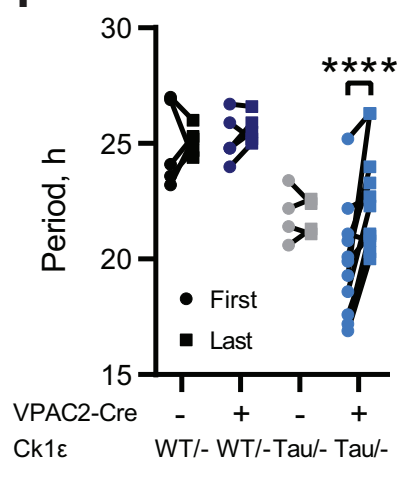

G

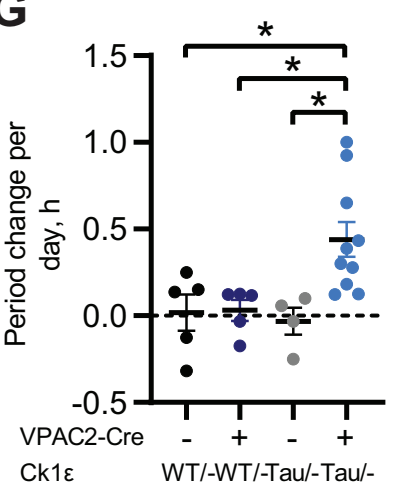

H

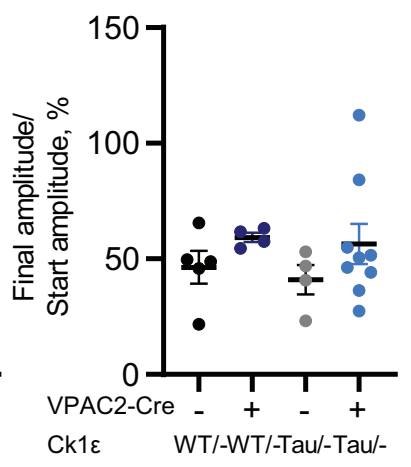

Figure 2. Circadian periods of bioluminescent rhythms of SCN slices from VPAC2-Cre/Ck1 $\varepsilon^{\text {Tau/- }}$ mice do not reflect behavioral period. $\boldsymbol{A}$, Representative baseline-corrected PER2::LUCIFERASE bioluminescence rhythms from $C k 1 \varepsilon^{\text {WT/- }}$, VPAC2-Cre/Ck1 $\varepsilon^{\text {WT/- }}, C k 1 \varepsilon^{\text {Tau/- }}$, and VPAC2-Cre/Ck1 $\varepsilon^{\text {Tau/- }} \mathrm{SCN}$ dissected following wheel-running recordings. B, Periods (mean \pm SEM) of the first 4 bioluminescent cycles of adult SCN slices as in $A$. C, Comparison of circadian periods of free-running behavior and slice bioluminescence rhythms (measured from the first 4 cycles) from individual mice. $\boldsymbol{D}$, Scatter plot of slice periods (measured from the first 4 cycles) versus behavioral periods. VPAC2-Cre/Ck1 $\varepsilon^{\text {Taul- }}$ mice show no significant correlation $(p=0.07$, Pearson's correlation), but grouped control slices do $\left(* * * * p<0.0001\right.$, Pearson's correlation). Lines indicate linear regression: VPAC2-Cre/Ck1 $\varepsilon^{\text {Tau } /-}, r^{2}=0.36, Y=0.1037 * X+21.25 ;$ Controls: $r^{2}=0.85$, $Y=0.5458 * X+10.47$. E, Representative baseline-corrected PER2::LUCIFERASE bioluminescence rhythms from C $k 1 \varepsilon^{\text {WT/ }}$ and VPAC2-Cre/Ck1 $\varepsilon^{\text {Taul- }}$ SCN slices displaying no phase alignment initially, followed by period lengthening in the VPAC2-Cre/Ck1 $\varepsilon^{\text {Tau- }}$ slice and resultant phase alignment. $\boldsymbol{F}$, Comparison of periods between the first two bioluminescent cycles (First) and final two cycles (Last) within each SCN slice. G, Period change per day (mean \pm SEM) in SCN slices. $\boldsymbol{H}$, Bioluminescence amplitude (mean \pm SEM) in the last 3 cycles as a percentage of the ampli-

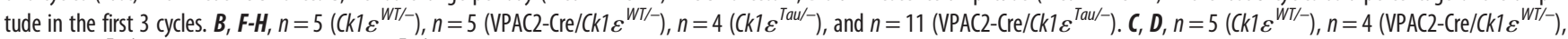
$n=4\left(C k 1 \varepsilon^{\text {Tau/ }}\right)$, and $n=10$ (VPAC2-Cre/Ck1 $\left.\varepsilon^{\text {Tau/- }}\right)$. *p $<0.05 ; * * * * p<0.0001$; two-way ANOVAs with Tukey's post hoc test.

penetrance in the corresponding SCN slices ex vivo. This variability was also evident in the stability of the individual SCN rhythms, whereby the period of VPAC2-Cre/Ck1 $\varepsilon^{\text {Tau/- }}$ slices tended to increase over time in culture (Fig. $2 E-G$; two-way ANOVA, $F_{(3,21)}=4.478, p=0.014$, interaction; Tukey's post hoc test $p<0.0001$ ), suggesting that the long-period VPAC2 cells were re-exerting influence over ensemble period. While there was considerable variability in the peak-to-peak period within the group (Fig. 2C,F), the average period increase was almost 30 min per day for VPAC2-Cre/Ck1 $\varepsilon^{\text {Tau/- }}$ slices, whereas control slices did not show significant period lengthening during ex vivo culture (Fig. 2G; two-way ANOVA, $F_{(1,20)}=4.445, p=0.048$, interaction; Tukey's post hoc test, $p=0.030$ vs $C k 1 \varepsilon^{W T /}$; $p=0.023$ vs $C k 1 \varepsilon^{\text {Taul- }} ; p=0.035$ vs VPAC2-Cre). Importantly, there was no significant difference in the progressive fall in amplitude observed over time in all genotypes (Fig. $2 \mathrm{H}$; two-way ANOVA, $F_{(1,18)}=2.555, p=0.13$, Cre; $F_{(1,18)}=0.216, p=0.65$, $\operatorname{Tau} ; F_{(1,18)}=0.020, p=0.89$, interaction), suggesting that the period lengthening was not the result of cells within the slice desynchronizing. Overall, we conclude that cell-autonomous properties of VPAC2 cells contribute strongly to computation of ensemble period in vivo, and also, but to a lesser extent, ex vivo.

The cell-autonomous clock of VPAC2 cells is essential for the circadian coordination of rest/activity rhythms

To test whether circadian competence in VPAC2 cells is essential for the generation of behavioral rhythms and/or molecular pacemaking in the SCN, we examined the impact of cell type-specific 
A

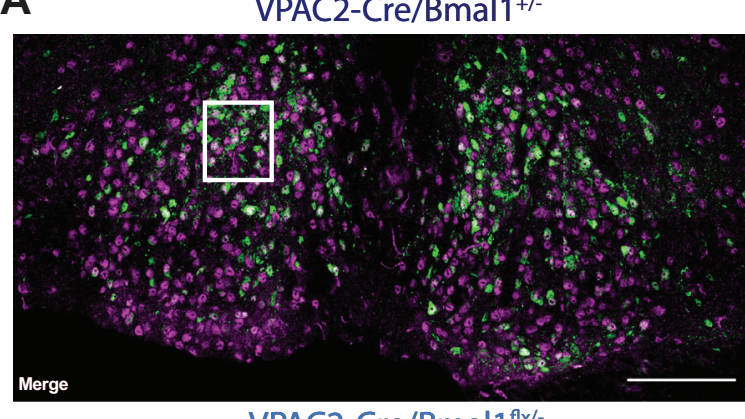

VPAC2-Cre/Bmal1 19x/

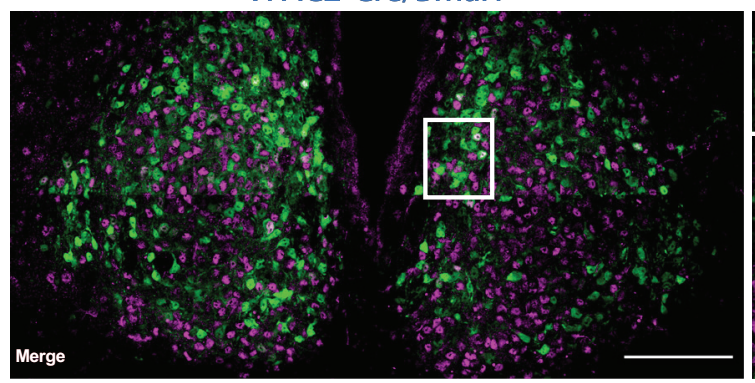

D

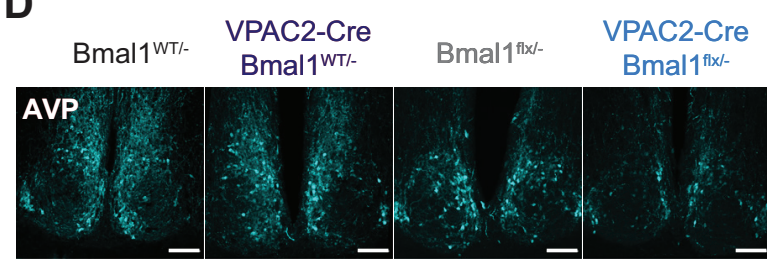

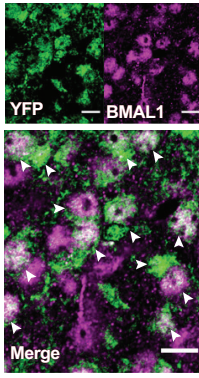

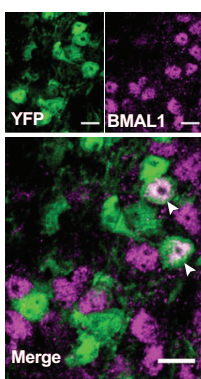

E
B

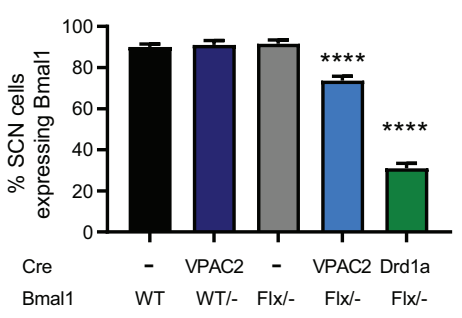

C

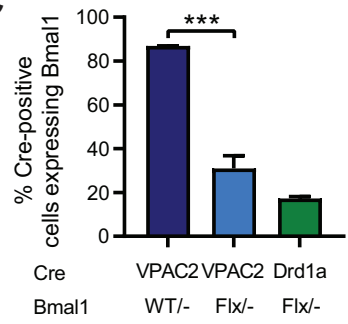

VIP

GRP

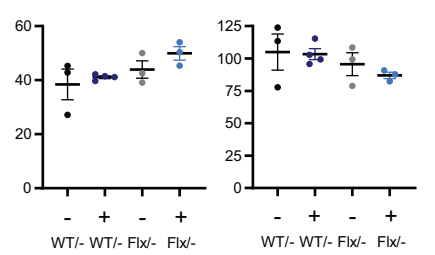

Figure 3. Targeted deletion of BMAL1 from VPAC2-Cre-expressing SCN cells. A, Representative $63 \times$ tiled confocal micrographs of Cre recombinase activity, as reported by a genetically encoded EYFP reporter (green), and BMAL1 immunohistochemistry (magenta) in VPAC2-Cre/Bmal1 ${ }^{W T /}$ and VPAC2-Cre/Bmal $7^{f / x /-}$ SCN sections. White rectangles represent locations of magnified images. White arrowheads indicate colocalization between EYFP and BMAL1-ir. Scale bars: stitched images, $100 \mu \mathrm{m}$; magnified images, $10 \mu \mathrm{m}$. $\boldsymbol{B}$, Percentage of SCN cells (marked by DAPI; mean \pm SEM) expressing BMAL1-ir across genotypes, including Drd1a-Cre/Bmal1 ${ }^{f f /}{ }^{-}$. C, Percentage of (re-positive cells (marked by EYFP; mean \pm SEM) expressing BMAL1-ir across genotypes. B, C, One-way ANOVA with Tukey's post hoc test. D, Representative images of AVP-ir in SCN of VPAC2-Cre/Bmal $7^{f f x /-}$ mice and controls. Scale bars, $100 \mu \mathrm{m}$. $\boldsymbol{E}$, Fluorescence intensities (mean \pm SEM) for immunohistochemical staining of AVP (left), VIP (middle), and GRP (right) in SCN sections of VPAC2-Cre/Bmal ${ }^{f f x-}$ mice and controls. B, C, $n=7$ (Bmal ${ }^{\text {WT/- }}$ ), $n=7$

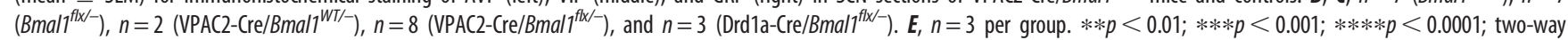
ANOVA with Tukey's post hoc test.

deletion of BMAL1 by Cre-mediated removal of a critical exon within a floxed allele of the Bmal1 gene (Fig. $1 A$ ). Homozygous loss of Bmal1 results in a severely disrupted or arrhythmic TTFL, although a single floxed copy of Bmall is fully functional and sufficient for normal pace-making (Bunger et al., 2000; Gibbs et al., 2012). All experiments were conducted using Bmal1 ${ }^{f l x /-}$ mice because the efficiency of Bmall deletion in the SCN is enhanced when the floxed Bmall allele is paired against a null allele (Husse et al., 2011). The efficiency of deletion in VPAC2expressing cells (constituting $\sim 35 \%$ of SCN cells) was determined by immunohistochemistry for BMAL1 expression and the expression of a genomic EYFP reporter (Rosa-LSL-EYFP) to identify Cre-expressing cells (Fig. 3A). VPAC2-Cre-mediated excision reduced the number of BMAL1-expressing cells across the SCN by $\sim 20 \%$ compared with WT SCN (Fig. 3B; one-way ANOVA, $F_{(4,22)}=98.83, p<0.0001$, Tukey's post hoc test, $p<0.0001$ ), which suggests appreciable but incomplete deletion from all VPAC2-expressing cells. Indeed, within the VPAC2-Cre-expressing cell population (labeled by EYFP), $\sim 30 \%$ of cells had detectable BMAL1-ir. This nevertheless emphasized the efficient and specific targeting of BMAL1 in $\sim 70 \%$ of VPAC2 cells (Fig. 3C). Importantly, the loss of BMAL1 from VPAC2-expressing cells did not affect overall
SCN morphology or expression of VIP or GRP (Fig. 3E). There was, however, a small $(\sim 30 \%)$ but significant decline in AVP-ir (Fig. 3D,E; two-way ANOVA, $F_{(1,9)}=14.34, p=0.004$, interaction; Sidak's post hoc $p=0.004$ vs Bmal1 $^{f l x /-}, p=0.002$ vs VPAC2Cre/Bmal1 $\left.{ }^{W T /-}\right)$, consistent with some cells coexpressing AVP and VPAC2 (Patton et al., 2020; Wen et al., 2020), and the Avp gene being a circadian clock-controlled target of the BMAL1-dependent TTFL (Jin et al., 1999; Mieda et al., 2015).

Mice of all genotypes (Bmal1 ${ }^{W T /-}$ and Bmal1 $1^{f l x /-}$ both without and with VPAC2-Cre) entrained stably under a 12:12 LD cycle with a period of $24 \mathrm{~h}$, and there were no obvious differences in activity patterns between them (Fig. 4A; two-way ANOVA, $F_{(1,29)}=0.459, p=0.5$, Cre; $F_{(1,29)}=0.672, p=0.42$, Bmall $; F_{(1,29)}=$ $2.81, p=0.10$, interaction). Mice were then transferred to DD for up to 7 weeks to allow the emergence of any $\mathrm{Bmal1}^{-/-}$-dependent phenotypes. Control mice (Bmal1 ${ }^{\text {WT/ }}$ with or without VPAC2-Cre, and Bmal1 ${ }^{f l x /-}$ without Cre) free-ran with clear circadian patterns and endogenous periods slightly longer than 24 h (Fig. $4 B, C$ ) and with well-defined amplitude (Fig. 4E). The VPAC2-Cre/Bmalf ${ }^{f l x /-}$ group, however, displayed highly variable phenotypes both between and within individual animals under DD (Fig. 4C). Thirteen of 18 mice showed a strong phenotype: arrhythmicity occurred in 5 mice, while fragmented or "split" 
behavior was the most common result, seen in 8 mice, with several animals displaying multiple significant periods (Fig. $4 B-D)$. Furthermore, in the majority of mice, the amplitude of the activity rhythm was reduced, leading to a significant difference with control groups (Fig. $4 E$; KruskalWallis test, $\mathrm{H}(3)=21.19, p<0.0001$, Dunn's post hoc $p<0.0001$ vs VPAC2Cre/Bmal1 ${ }^{W T /-} ; p=0.032$ vs Bmal1 ${ }^{f l x /-}$ ). Strikingly, BMAL1-dependent phenotypes (arrhythmicity, multiple periodicities, loss of amplitude) did not appear at a consistent point following transfer to DD. Rather, they accumulated progressively (Fig. $4 F$ ), at a range of times throughout the experiment: some mice immediately showed split or arrhythmic behavior, whereas others took several weeks and displayed phenotypes that looked WT until that point. We conclude that the cell-autonomous rhythmicity of VPAC2-expressing cells is essential for normal circadian control of behavior, although considerable plasticity exists within and between individual mice that affects the precise timing and presentation of the VPAC2Cre/Bmal1 ${ }^{f l x}$ phenotype.

To determine whether the behavioral effects of the local loss of BMAL1 in VPAC2 cells were specific to that cell type, an additional group of mice was included in which BMAL1 was deleted from cells expressing Cre driven by the Drdla promoter. In these animals, BMAL1 was deleted in $\sim 80 \%$ of Drdla-specific cells compared with $\sim 70 \%$ of VPAC2-expressing cells in VPAC2-Cre/Bmal1 ${ }^{f l x /-}$ mice (Fig. $3 C$ ), resulting in an overall deletion of BMAL1 across $\sim 70 \%$ of total SCN cells in Drdla-Cre/Bmal1 ${ }^{f l x /}$ mice (Fig. $3 B$; one-way ANOVA, $\quad F_{(4,22)}=98.83, p<0.0001$, Tukey's post hoc test, $p<0.0001$ vs Bmal1 $^{\text {WT/- }}$ or VPAC2-Cre/Bmal1 ${ }^{f l x /-}$ ). Notwithstanding this broader deletion of BMAL1, the rest/activity rhythms of these mice were comparable to those of the control groups: only 3 of 7 showed minor instability in the time of onset of wheelrunning, but none of them showed a phenotype comparable to those of VPAC2Cre/Bmall ${ }^{f x /-}$ mice (Fig. $4 G$ ), and the RAs of their rhythms were not significantly different from control groups (Fig. $4 E$; Dunn's post hoc $p>0.9999$ vs Bmal1 $1^{f l x /-}$ ). These results emphasize that the effects of deletion of BMAL1 from VPAC2 cells are not merely because of reaching a threshold number of SCN neurons, given that the Drdla-Cre-mediated removal targeted over twice as many cells as that of VPAC2-Cre. Rather, they suggest that the nature and identity of the cells from which BMAL1 is deleted (in this case VPAC2 or Drd1a) are paramount.
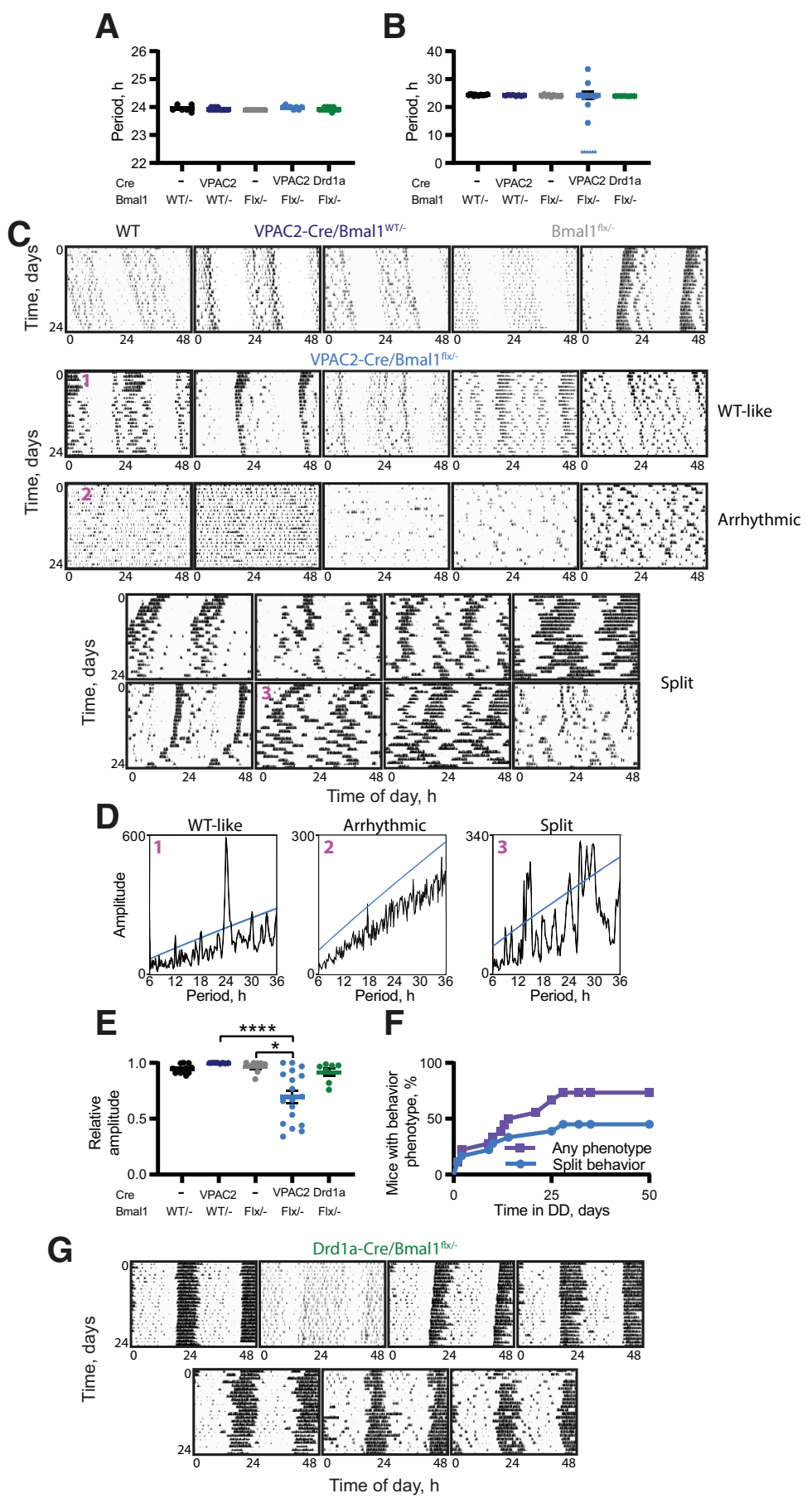

Figure 4. Deletion of BMAL1 from VPAC2-Cre-expressing cells compromises circadian behavior. $\boldsymbol{A}, \boldsymbol{B}$, Circadian periods (mean \pm SEM) of VPAC2-Cre/Bmal $7^{f \mathrm{fl} /-}$ mice and controls under (A) 12:12 LD and (B) DD. For 6 VPAC2-Cre/Bmal ${ }^{f(x)-}$ mice, an explicit single period could not be determined because of their disturbed behavior; and so, these are added as stars as a nominal $3 \mathrm{~h}$, and were excluded from statistical analysis. Two-way ANOVA (excluding Drd1a-Cre/Bmal $7^{f f / r}$ mice); one-way ANOVA, including all groups for Drd1a-Cre/Bmal $7^{f \times /-}$ comparison. C, Representative double-plotted actograms of final $24 \mathrm{~d}$ of

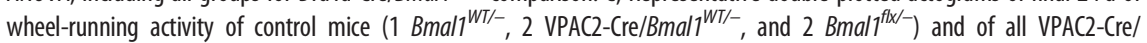
$B$ mal $7^{f(x /-}$ mice. VPAC2-Cre/Bmalf ${ }^{f(x /-}$ actograms are divided into three groups (WT-like, Arrhythmic, and Split) and then ranked in descending order of RA score under DD (final $14 \mathrm{~d}$ of recording). $\boldsymbol{D}, \chi^{2}$ periodograms for $3 \mathrm{VPAC2}$-Cre/Bmal $7^{f(x)-}$ as indicated in $\mathbf{C}: \mathbf{D 1}$, has a single significant peak at $\sim 24 \mathrm{~h} ; \mathbf{D 2}$, has no significant period; $\mathbf{D}$, displays multiple significant periods. $E$, RA scores (mean \pm SEM) from the last $14 \mathrm{~d}$ of wheel-running activity under DD. Kruskal-Wallis test with Dunn's

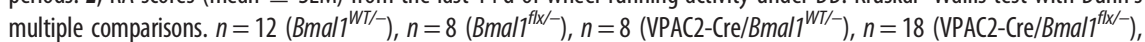
and $n=7$ (Drd1a-Cre/Bmal $7^{f(x)-}$ ). F, Percentage of VPAC2-Cre/Bmal $7^{f(x)-}$ mice displaying behavioral phenotypes (purple, squares) or specifically split behavior (blue, circles) over time. G, Double-plotted actograms of wheel-running activity of all Drd1a-Cre/Bmal $7^{f(x /-}$ mice. $* p<0.05 . * * * * p<0.0001$. 
The cell-autonomous clock of VPAC2 cells is essential for molecular pace-making in the SCN

The loss of behavioral rhythms in conditionally BMAL1-deleted mice could indicate that a functional cell-autonomous TTFL in VPAC2 cells is required for either producing a coherent ensemble signal within the SCN circuit or for enabling distribution of an appropriate output signal from the SCN to relevant brain centers. To test this, SCN slices were prepared from adult mice following the recording of wheel-running rhythms to establish whether behavioral phenotypes were reflected by, and thus likely resulted from, VPAC2-mediated changes in SCN rhythmicity. Circadian rhythms of PER2::LUC bioluminescence from slices lacking either the floxed Bmal1 allele or VPAC2-Cre were stable and of high amplitude (Fig. 5A,B). In contrast, VPAC2-Cre/ $B$ mal1 ${ }^{f l x /}$ SCN slices were highly disorganized, showing erratic and unstable bioluminescence (Fig. 5A), and having significantly higher RAE scores compared with controls (Fig. 5C; one-way ANOVA, $F_{(4,32)}=8.147, p=0.0001$, Tukey's post hoc test, $p=0.005$ vs Bmal1 ${ }^{f l x /} ; p=0.0004$ vs VPAC2Cre/Bmal1 ${ }^{W T /-}$ ). The circadian behavior of VPAC2-Cre/Bmal1 ${ }^{f l x /-}$ mice was therefore directly reflected in the lack of competence of molecular pace-making of the TTFL SCN rhythm: a disrupted, unstable behavioral rhythm was predictive of a poorly organized rhythm in the SCN from the same animal (Fig. 5D). In contrast to the disruption following deletion of BMAL1 from VPAC2 cells, but consistent with minor behavioral effects, the more extensive Drdla-Cre-mediated deletion of BMAL1 had little impact on the SCN rhythms. They were well defined (Fig. $5 A$ ) with a period (Fig. $5 B$; one-way ANOVA, $F_{(4,32)}=1.425, p=0.93$ ) and a low RAE score comparable to those of the control groups (Fig. $5 C$; Tukey's post hoc test, $p=0.77$ vs Bmal1 $1^{f x /-}, p=0.54$ vs Bmal1 ${ }^{W T /-}$ ). The competence of their SCN was therefore consistent with their well-organized wheel-running behavior. These results confirm the specificity of the contribution of VPAC2-expressing cells to the ensemble rhythm and circadian behavior, whereby the ablation of the cell-autonomous clock of VPAC2 cells is sufficient to abrogate SCN, and thence behavioral, rhythmicity. This highlights VPAC2expressing cells as necessary determinants of SCN circadian output and behavioral regulation.

\section{Discussion}

The potency of the SCN as circadian pacemaker arises from network-level interactions that confer on it the emergent properties essential to its role. The SCN is, however, a heterogeneous mix of neuropeptidergic cell types, and so identifying the contribution(s) of defined cell populations to circuit-level function(s) is an important problem in circadian neurobiology. Here, we used intersectional genetics to manipulate the cell-autonomous clockwork of cells that express the VPAC2 receptor for VIP, and thereby demonstrated that these cells control circadian behavior and pacemaking. These cells therefore constitute an important element of the network topology of the SCN, acting downstream of retinal and VIP-mediated signaling to maintain local and distant circadian coherence.

The ability of VPAC2 cells to determine behavioral circadian period complements other intersectional studies. Whereas lengthening the period of VIP cells (Vip-Clock ${ }^{\Delta 19}$ ) had no effect on free-running rhythmicity, the same manipulation in neuromedin S (NMS) cells (NMS-Clock ${ }^{\Delta 19}$ ), including almost all VIP and AVP cells, lengthened the behavioral period (Lee et al., 2015). Similarly, period lengthening in Drdla-Cre cells (including $\sim 60 \%$ of AVP cells) in Drd1a-Cre/Ck1 $\varepsilon^{\text {Tau/Tau }}$ mice
A
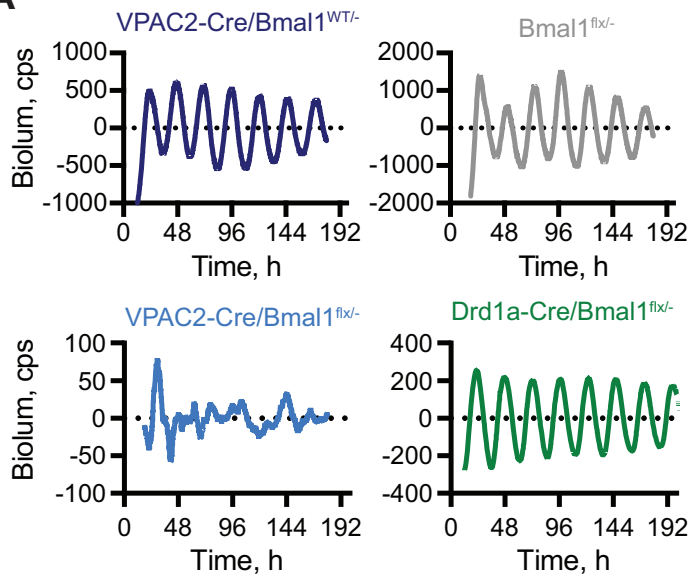

B
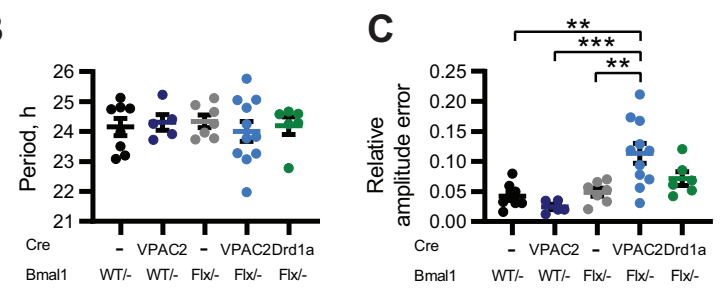

D
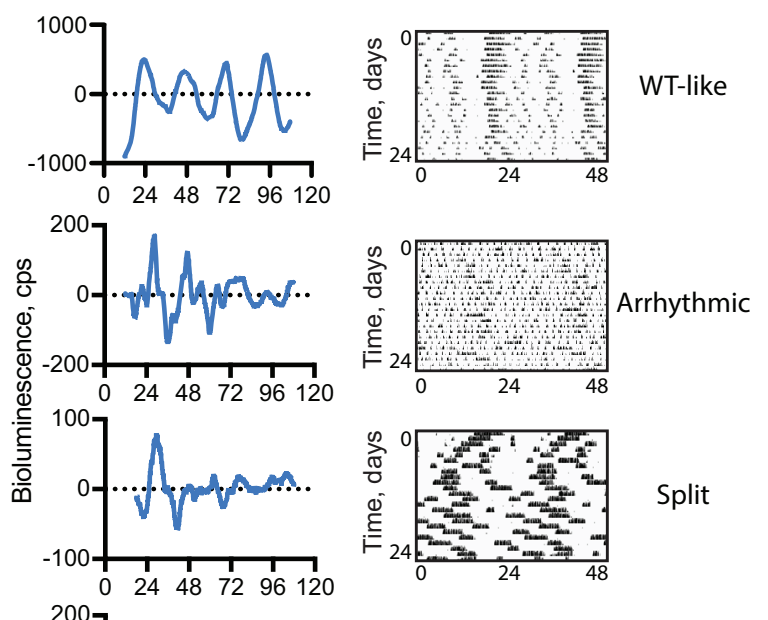

Split
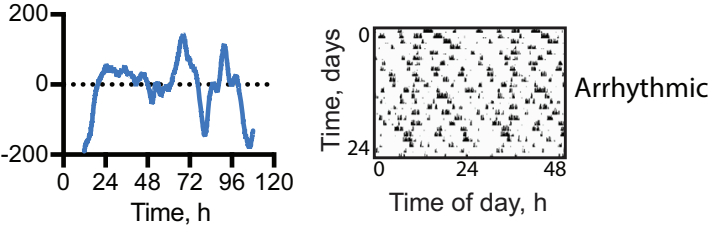

Figure 5. Deletion of BMAL1 from VPAC2-Cre-expressing cells compromises SCN molecular pace-making. $\boldsymbol{A}$, Representative, baseline-corrected PER2:::LUCIFERASE bioluminescence traces from control, VPAC2-Cre/Bmal $7^{f(x /-}$, and Drd1a-Cre/Bmal ${ }^{f(x /}-$ SCN dissected in dim red light following recording of wheel-running rhythms in DD. $B$, Period (mean \pm SEM) of the first 4 bioluminescent cycles recorded from adult SCN slices; genotypes as in $A . n=8\left(B m a l 7^{W T /}\right)$,

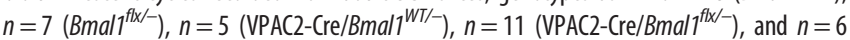
(Drd1a-Cre/Bmal $\left.{ }^{f(x)-}\right)$. C, RAE scores (mean \pm SEM) for bioluminescent recordings from SCN slices. $n$ values same as in $\boldsymbol{B}$. $\boldsymbol{D}$, Representative PER2::LUCIFERASE bioluminescence rhythms from VPAC2-Cre/Bmalf $7^{f(x /-}$ SCN slices alongside respective actograms from corresponding mice. "WT-like," "Arrhythmic," or "Split" beside the actograms indicates the phenotypic category. $* * p<0.01 ; * * * p<0.001$; one-way ANOVA, with Tukey's post hoc test.

lengthened their behavioral period (Smyllie et al., 2016). Finally, directly altering the period of AVP cells alone accordingly shortened or lengthened behavioral rhythms (Mieda et al., 2016). Given that VPAC2 cells encompass $\sim 85 \%$ of AVP cells, the current period effects likely arise from altering cell-autonomous 
oscillators of VPAC2/AVP cells. Moreover, comparable effects are seen when targeting AVP or VPAC2 cells ex vivo, insofar as the SCN period does not recapitulate behavioral periods. For example, in vivo period lengthening of AVP cells to $26 \mathrm{~h}$ disappeared after the first cycle ex vivo, when SCN slices reverted to a WT 24 h (Mieda et al., 2016). Ex vivo, SCN AVP cells are therefore less potent in period setting than in vivo, similar to VPAC2targetted SCN. Interestingly, however, VPAC2-Cre/Ck1 $\varepsilon^{\text {Tau/- }}$ slices showed a progressive lengthening of period, not seen in control slices, without loss of precision or amplitude. This suggests that, with extended culture, the longer-period VPAC2 cells gained influence over the ensemble period, indicative of circuitlevel plasticity. Indeed, the circuit context in which VPAC2 cells are embedded appears to be essential for period determination. A recent study found that VPAC2 cells additionally required the contribution of VIP cells to dictate SCN slice period (Patton et al., 2020); and, given both their shell localization and likely expression of glutamate receptor NR2C (Wen et al., 2020), they may also act as a conduit for astrocytically derived period cues (Brancaccio et al., 2017).

Overall, therefore, the ability of VPAC2/AVP cells to determine circadian period is greatly enhanced in the intact animal compared with slices. While slice period variability may arise from technical factors, such as Cre excision efficiency, the marked, consistent behavioral effect confirmed effective deletion. It is possible that VPAC2 cells function primarily as output cells of the SCN rather than dictating SCN periodicity per se, so their cell-intrinsic period is reflected at the behavioral level but not in slices. This would, however, require two separately oscillating populations within the SCN, which would be expected to lead to more unstable behavioral patterns than were observed. Instead, it is likely that modification of SCN function by behavioral state feedback, at the circuit level or directly to AVP and/or VPAC2 cells, underlies these differences. A precedent for behavioral feedback potentiating a compromised SCN is seen in arrhythmic VPAC2-null mice, which, after entrainment to a $24 \mathrm{~h}$ schedule of voluntary exercise, exhibit free-running circadian behavior on release into DD (Power et al., 2010). For VPAC2-Cre/Ck1 $\varepsilon^{\text {Tau/- }}$ mice, sparse VPAC2 cells in the rest of the hypothalamus that receive input from the $\mathrm{SCN}$, such as in the paraventricular nucleus (Kalló et al., 2004), would also have $24 \mathrm{~h}$ periods and may feedback to, and resonate with, the intrinsically $24 \mathrm{~h}$ SCN VPAC2 cells. This feedback would reinforce the role of SCN VPAC2 cells in period determination in vivo, leading to a change in behavior, but would be absent in slices.

In addition to period setting, VPAC2 cells are also critical for rhythm maintenance, as evidenced by VPAC2-specific BMAL1 deletion. Interestingly, the total number of targetted cells appears to be less important than the type of cell rendered circadian incompetent. Loss of BMAL1 from 30\% of SCN neurons using Nestin-Cre had no effect on behavioral rhythms in DD (Mieda and Sakurai, 2011). Equally, deletion of BMAL1 in $65 \%$ of SCN cells using heterozygous Synaptotagmin10-Cre had no behavioral effect (Husse et al., 2011). Only in homozygous Synaptotagmin10-Cre mice, where BMAL1 was deleted from $83 \%$ of the SCN, was a behavioral phenotype evident. Moreover, in the current study, loss of BMAL1 from Drd1aexpressing cells, causing $\sim 70 \%$ loss of BMAL1 across the SCN, did not compromise circadian behavior. Conversely, deletion of BMAL1 from NMS neurons, constituting $40 \%$ of the SCN, caused behavioral arrhythmia. In the current study, loss of BMAL1 from only $15 \%-20 \%$ of total SCN cells, but $70 \%$ of VPAC2 cells, severely disrupted circadian behavior. Unlike in pace-making, where VPAC2 and AVP share many commonalities, loss of BMAL1 exclusively from AVP cells did not cause disrupted circadian behavior, instead causing the nocturnal activity profile to widen and overall period to lengthen (Mieda et al., 2015; Shan et al., 2020). Thus, VPAC2 cells appear to constitute a population in which rhythmicity is essential for overall circadian competence.

Further demonstration of the essential role of VPAC2 cells came from ex vivo SCN slices, which exhibited highly disordered oscillations of TTFL activity, commensurate with loss of behavioral coherence. This contrasts with the sustained TTFL function following the ablation of BMAL1 from Drd1a-Cre cells in the current study, and solely from AVP cells, in which network synchrony is disrupted but ensemble rhythmicity is sustained through non-AVP cell oscillations (Mieda et al., 2015; Shan et al., 2020). This suggests that the loss of behavioral coherence seen in VPAC2-Cre/Bmal $1^{f l x /-}$ mice has a more fundamental origin than simply an inability to convey behaviorally relevant circadian cues outside the SCN (as in Prok2 receptor KO) (Prosser et al., 2007). Even more strikingly, Ko et al. (2010) demonstrated that global Bmal1 ${ }^{-1-}$ slices show stochastic quasi-circadian rhythmicity; thus, it is remarkable that loss of BMAL1 from $20 \%$ of the SCN caused such a pronounced phenotype. This provides strong evidence that VPAC2 cells in the SCN exert powerful control over ensemble rhythmicity, a result complementing rhythmicity restoration experiments of Patton et al. (2020), together showing that a competent TTFL in VPAC2 cells is necessary for coherent circadian oscillation.

Notwithstanding the overall effect of BMAL1 deletion, it is clear that the behavioral phenotype of VPAC2-Cre/Bmaly $1^{f l x /-}$ mice was variable both in severity and latency of presentation under DD, suggesting that there are subpopulations of VPAC2 cells, as posited for other SCN populations (Kawamoto et al., 2003; Geoghegan and Carter, 2008). The advent of single-cell transcriptomic profiling (Park et al., 2016; Wen et al., 2020) may reveal suitable markers for such subpopulations. That the behavioral disruption took time to emerge is intriguing, because, as with $N m s$-Bmal1 ${ }^{f l x / f l x}$ mice, phenotypes often appeared suddenly, with no obvious trigger, suggesting "the presence of mechanisms capable of transiently compensating for the loss of molecular clocks" (Lee et al., 2015). The initial rhythmicity in vivo, perhaps initiated by daily lighting cycles and/or sustained by light-driven behavioral rhythms, may have relied on coupling effects of nonVIPergic cells that compensated for the loss of VPAC2 cell-autonomous oscillators. Potential compensatory factors are AVP and GRP, both of which can induce rhythmicity in VIP- or VPAC2-deficient SCN (Brown et al., 2005; Maywood et al., 2006, 2011). Levels of AVP, however, were reduced in VPAC2-Cre/ Bmal1 ${ }^{f x /}$ mice, which may have exacerbated circadian disorganization arising from loss of TTFL function in VPAC2 cells. The signaling molecules used by VPAC2 cells themselves remain unclear, not least because a global AVP KO has only a minor effect on rhythmicity (Groblewski et al., 1981), although loss of AVP receptors does compromise intercellular coupling in the SCN (Yamaguchi et al., 2013). Similarly, NMS, another peptide marker for both core and shell SCN, is not essential for circadian function (Lee et al., 2015).

In conclusion, we have demonstrated that VPAC2 cells constitute an essential pace-setting and rhythm-generating population of the mammalian circadian system, necessary for fully coherent circadian oscillation both in vivo and ex vivo. Furthermore, we found that the strong pace-setting abilities of VPAC2 cells are disrupted, albeit not completely 
absent, on slice preparation, suggesting an unanticipated influence of extra-SCN populations in reinforcing periodicity. These findings complement those of Patton et al. (2020) that circadian competence in both VPAC2 cells and VIP cells is necessary for the de novo initiation of ensemble rhythms in SCN slices and behavior. Thus, circadian competence in VPAC2 cells is necessary for rhythmicity, but they require their cognate signaling cell partners to be sufficient for rhythmicity. The VIPergic axis confers much of the fundamental robustness and intercellular communication that is essential to normal SCN function, and our work advances our knowledge of the individual functions of the cells inherent to this axis.

\section{References}

Abrahamson EE, Moore RY (2001) Suprachiasmatic nucleus in the mouse: retinal innervation, intrinsic organization and efferent projections. Brain Res 916:172-191.

Antle MC, Silver R (2005) Orchestrating time: arrangements of the brain circadian clock. Trends Neurosci 28:145-151.

Aton SJ, Colwell CS, Harmar AJ, Waschek J, Herzog ED (2005) Vasoactive intestinal polypeptide mediates circadian rhythmicity and synchrony in mammalian clock neurons. Nat Neurosci 8:476-483.

Brancaccio M, Patton AP, Chesham JE, Maywood ES, Hastings MH (2017) Astrocytes control circadian timekeeping in the suprachiasmatic nucleus via glutamatergic signaling. Neuron 93:1420-1435.e5.

Brown TM, Hughes AT, Piggins HD (2005) Gastrin-releasing peptide promotes suprachiasmatic nuclei cellular rhythmicity in the absence of vasoactive intestinal polypeptide-VPAC2 receptor signaling. J Neurosci 25:11155-11164.

Bunger MK, Wilsbacher LD, Moran SM, Clendenin C, Radcliffe LA, Hogenesch JB, Simon MC, Takahashi JS, Bradfield CA (2000) Mop3 is an essential component of the master circadian pacemaker in mammals. Cell 103:1009-1017.

Ciarleglio CM, Gamble KL, Axley JC, Strauss BR, Cohen JY, Colwell CS, McMahon DG (2009) Population encoding by circadian clock neurons organizes circadian behavior. J Neurosci 29:1670-1676.

Colwell CS, Michel S, Itri J, Rodriguez W, Tam J, Lelievre V, Hu Z, Liu X, Waschek JA (2003) Disrupted circadian rhythms in VIP- and PHI-deficient mice. Am J Physiol Regul Integr Comp Physiol 285:R939-R949.

Geoghegan D, Carter DA (2008) A novel site of adult doublecortin expression: neuropeptide neurons within the suprachiasmatic nucleus circadian clock. BMC Neurosci 9:2-9.

Gibbs JE, Blaikley J, Beesley S, Matthews L, Simpson KD, Boyce SH, Farrow SN, Else KJ, Singh D, Ray DW, Loudon AS (2012) The nuclear receptor $\mathrm{REV}-\mathrm{ERB} \alpha$ mediates circadian regulation of innate immunity through selective regulation of inflammatory cytokines. Proc Natl Acad Sci USA 109:582-587.

Groblewski TA, Nunez AA, Gold RM (1981) Circadian rhythms in vasopressin deficient rats. Brain Res Bull 6:125-130.

Hamnett R, Crosby P, Chesham JE, Hastings MH (2019) Vasoactive intestinal peptide controls the suprachiasmatic circadian clock network via ERK1/2 and DUSP4 signalling. Nat Commun 10:542.

Harmar AJ, Marston HM, Shen S, Spratt C, West KM, Sheward WJ, Morrison CF, Dorin JR, Piggins HD, Reubi JC, Kelly JS, Maywood ES, Hastings MH (2002) The VPAC2 receptor is essential for circadian function in the mouse suprachiasmatic nuclei. Cell 109:497-508.

Hastings MH, Reddy AB, McMahon DG, Maywood ES (2005) Analysis of circadian mechanisms in the suprachiasmatic nucleus by transgenesis and biolistic transfection. Methods Enzymol 393:579-592.

Hastings MH, Maywood ES, Brancaccio M (2018) Generation of circadian rhythms in the suprachiasmatic nucleus. Nat Rev Neurosci 19:453-469.

Husse J, Zhou X, Shostak A, Oster H, Eichele G (2011) Synaptotagmin 10Cre, a driver to disrupt clock genes in the SCN. J Biol Rhythms 26:379389.

Jin X, Shearman LP, Weaver DR, Zylka MJ, De Vries GJ, Reppert SM (1999) A molecular mechanism regulating rhythmic output from the suprachiasmatic circadian clock. Cell 96:57-68.
Jones JR, Simon T, Lones L, Herzog ED (2018) SCN VIP neurons are essential for normal light-mediated resetting of the circadian system. J Neurosci 38:7986-7995.

Kalló I, Kalamatianos T, Wiltshire N, Shen S, Sheward W, Harmar AJ, Coen CW (2004) Transgenic approach reveals expression of the VPAC2 receptor in phenotypically defined neurons in the mouse suprachiasmatic nucleus and in its efferent target sites. Eur J Neurosci 19:2201-2211.

Kawamoto K, Nagano M, Kanda F, Chihara K, Shigeyoshi Y, Okamura H (2003) Two types of VIP neuronal components in rat suprachiasmatic nucleus. J Neurosci Res 74:852-857.

Ko CH, Yamada YR, Welsh DK, Buhr ED, Liu AC, Zhang EE, Ralph MR, Kay SA, Forger DB, Takahashi JS (2010) Emergence of noise-induced oscillations in the central circadian pacemaker. PLoS Biol 8:e1000513.

Krempels K, Usdin TB, Harta G, Mezey E (1995) PACAP acts through VIP type 2 receptors in the rat testis. Neuropeptides 29:315-320.

Lee IT, Chang AS, Manandhar M, Shan Y, Fan J, Izumo M, Ikeda Y, Motoike T, Dixon S, Seinfeld JE, Takahashi JS, Yanagisawa M (2015) Neuromedin S-producing neurons act as essential pacemakers in the suprachiasmatic nucleus to couple clock neurons and dictate circadian rhythms. Neuron 85:1086-1102.

Liu AC, Welsh DK, Ko CH, Tran HG, Zhang EE, Priest AA, Buhr ED, Singer O, Meeker K, Verma IM, Doyle FJ, Takahashi JS, Kay SA (2007) Intercellular coupling confers robustness against mutations in the SCN circadian clock network. Cell 129:605-616.

Luo L, Ambrozkiewicz MC, Benseler F, Chen C, Dumontier E, Falkner S, Furlanis E, Gomez AM, Hoshina N, Huang WH, Hutchison MA, ItohMaruoka Y, Lavery LA, Li W, Maruo T, Motohashi J, Pai EL, Pelkey KA, Pereira A, Philips T, et al. (2020) Optimizing nervous system-specific gene targeting with Cre driver lines: prevalence of germline recombination and influencing factors. Neuron 106:37-29.

Maywood ES, Reddy AB, Wong GK, O’Neill JS, O’Brien JA, McMahon DG, Harmar AJ, Okamura H, Hastings MH (2006) Synchronization and maintenance of timekeeping in suprachiasmatic circadian clock cells by neuropeptidergic signaling. Curr Biol 16:599-605.

Maywood ES, Chesham JE, O’Brien JA, Hastings MH (2011) A diversity of paracrine signals sustains molecular circadian cycling in suprachiasmatic nucleus circuits. Proc Natl Acad Sci USA 108:14306-14311.

Mazuski C, Abel JH, Chen SP, Hermanstyne TO, Jones JR, Simon T, Doyle FJ, Herzog ED (2018) Entrainment of circadian rhythms depends on firing rates and neuropeptide release of VIP SCN neurons. Neuron 99:555563.

McDearmon EL, Patel KN, Ko CH, Walisser JA, Schook AC, Chong JL, Wilsbacher LD, Song EJ, Hong HK, Bradfield CA, Takahashi JS (2006) Dissecting the functions of the mammalian clock protein BMAL1 by tissue-specific rescue in mice. Science 314:1304-1308.

Meng QJ, Logunova L, Maywood ES, Gallego M, Lebiecki J, Brown TM, Sládek M, Semikhodskii AS, Glossop NR, Piggins HD, Chesham JE, Bechtold DA, Yoo SH, Takahashi JS, Virshup DM, Boot-Handford RP, Hastings MH, Loudon AS (2008) Setting clock speed in mammals: the $\mathrm{CK} 1 \varepsilon$ tau mutation in mice accelerates circadian pacemakers by selectively destabilizing PERIOD proteins. Neuron 58:78-88.

Mieda M, Sakurai T (2011) Bmall in the nervous system is essential for normal adaptation of circadian locomotor activity and food intake to periodic feeding. J Neurosci 31:15391-15396.

Mieda M, Ono D, Hasegawa E, Okamoto H, Honma K, Honma S, Sakurai T (2015) Cellular clocks in AVP neurons of the SCN are critical for interneuronal coupling regulating circadian behavior rhythm. Neuron 85:1103-1116.

Mieda M, Okamoto H, Sakurai T (2016) Manipulating the cellular circadian period of arginine vasopressin neurons alters the behavioral report. Curr Biol 26:1-8.

Park J, Zhu H, O'Sullivan S, Ogunnaike BA, Weaver DR, Schwaber JS, Vadigepalli R (2016) Single-cell transcriptional analysis reveals novel neuronal phenotypes and interaction networks involved in the central circadian clock. Front Neurosci 10:1-19.

Patton AP, Chesham JE, Hastings MH (2016) Combined pharmacological and genetic manipulations unlock unprecedented temporal elasticity and reveal phase-specific modulation of the molecular circadian clock of the mouse suprachiasmatic nucleus. J Neurosci 36:9326-9341.

Patton AP, Edwards MD, Smyllie NJ, Hamnett R, Chesham JE, Brancaccio M, Maywood ES, Hastings MH (2020) The VIP-VPAC2 neuropeptidergic axis 
is a cellular pace-making hub of the suprachiasmatic nucleus circadian circuit. Nat Commun 11:3394.

Power A, Hughes AT, Samuels RE, Piggins HD (2010) Rhythm-promoting actions of exercise in mice with deficient neuropeptide signaling. J Biol Rhythms 25:235-246.

Prosser HM, Bradley A, Chesham JE, Ebling FJ, Hastings MH, Maywood ES (2007) Prokineticin receptor 2 (Prokr2) is essential for the regulation of circadian behavior by the suprachiasmatic nuclei. Proc Natl Acad Sci USA 104:648-653.

Reddy AB, Karp NA, Maywood ES, Sage EA, Deery M, O'Neill JS, Wong GK, Chesham J, Odell M, Lilley KS, Kyriacou CP, Hastings MH (2006) Circadian orchestration of the hepatic proteome. Curr Biol 16:11071115.

Reppert SM, Weaver DR (2002) Coordination of circadian timing in mammals. Nature 418:935-941.

Shan Y, Abel JH, Li Y, Izumo M, Cox KH, Jeong B, Yoo SH, Olson DP, Doyle FJ, Takahashi JS (2020) Dual-color single-cell imaging of the suprachiasmatic nucleus reveals a circadian role in network synchrony. Neuron 108:164-179.e7.

Smyllie NJ, Chesham JE, Hamnett R, Maywood ES, Hastings MH (2016) Temporally chimeric mice reveal flexibility of circadian period setting in the suprachiasmatic nucleus. Proc Natl Acad Sci USA 113:3657-3662.
Srinivas S, Watanabe T, Lin CS, William CM, Tanabe Y, Jessell TM, Costantini F (2001) Cre reporter strains produced by targeted insertion of EYFP and ECFP into the ROSA26 locus. BMC Dev Biol 1:4.

Usdin T, Bonner T, Mezey E (1994) Two receptors for vasoactive intestinal polypeptide with similar specificity and complementary distributions. Endocrinology 135:2662-2680.

Wen S, Ma D, Zhao M, Xie L, Wu Q, Gou L, Zhu C, Fan Y, Wang H, Yan J (2020) Spatiotemporal single-cell analysis of gene expression in the mouse suprachiasmatic nucleus. Nat Neurosci 23:456-467.

Yamaguchi Y, Suzuki T, Mizoro Y, Kori H, Okada K, Chen Y, Fustin JM, Yamazaki F, Mizuguchi N, Zhang J, Dong X, Tsujimoto G, Okuno Y, Doi M, Okamura H (2013) Mice genetically deficient in vasopressin V1a and $\mathrm{V} 1 \mathrm{~b}$ receptors are resistant to jet lag. Science 342:85-90.

Yoo SH, Yamazaki S, Lowrey PL, Shimomura K, Ko CH, Buhr ED, Siepka SM, Hong HK, Oh WJ, Yoo OJ, Menaker M, Takahashi JS (2004) PERIOD2::LUCIFERASE real-time reporting of circadian dynamics reveals persistent circadian oscillations in mouse peripheral tissues. Proc Natl Acad Sci USA 101:5339-5346.

Zielinski T, Moore AM, Troup E, Halliday KJ, Millar AJ (2014) Strengths and limitations of period estimation methods for circadian data. PLoS One 9:e96462. 